\title{
Triplet exciton transport in isotopic mixed naphthalene crystals. II. Master equation analysis ${ }^{a)}$
}

\author{
Stuart T. Gentry ${ }^{\text {b) }}$ and Raoul Kopelman \\ Department of Chemistry, The University of Michigan, Ann Arbor, Michigan 48109
}

(Received 16 November 1983; accepted 15 May 1984)

\begin{abstract}
The experimental data on triplet exciton transport in isotopically mixed crystals of naphthalene/ perdeuteronaphthalene (paper I) are contrasted with singlet exciton transport in the same samples (20\%-100\%) and analyzed in terms of incoherent hopping models. The master equation approach is emphasized and extended. We modify the conventional continuum master equations via a physically plausible cutoff of the high-frequency transfer rates. This results in an experimentally acceptable functional form (transport linear with high power of concentration) and nearest-neighbor transfer time (100 ps). We also developed a lattice master equation (numerically soluble), using an experimentally tested exciton superexchange formula. The somewhat surprising result is that the lattice master equations do not fit the experimental functional form. The success of the continuum models and the failure of the lattice models are attributed to the latter's neglect of the spread in transfer rates for a given intersite distance. We claim that clusterization as well as diagonal homogeneous and/or inhomogeneous disorder cause the above spread. On the other hand, these energy mismatches are small with respect to the thermal energy, in contrast to the singlet exciton transport case, where, due to larger energy mismatches, a percolation-like critical concentration is observed. Thus for the given concentration and temperature regimes, the triplet exciton transport is diffusive while the singlet exciton transport is percolative. Lower temperatures and/or concentrations are required for percolative triplet energy transport in these systems.
\end{abstract}

\section{INTRODUCTION}

Energy transport in disordered media is an interesting, but complex, topic. It is a relatively straightforward process to solve for exciton transport in the limit of a translationally invariant lattice ${ }^{1-3}$ or in the limit of a lattice with dilute guest (i.e., conducting) sites. ${ }^{4-6}$ The intermediate case of moderate guest site concentrations is much more difficult to analyze. Varying the guest concentration influences exciton transport via several mechanisms. The major one is that changing the average guest site separation distance affects the rates of the pairwise transfer steps and consequently the overall transport rate. We will demonstrate that, in addition to this effect, the concentration dependent variation in the local environment around the guest sites (e.g., clusterization) also has a significant influence on exciton transport in isotopically mixed naphthalene crystals. We will base this conclusion on the analysis of experimental triplet exciton transport data using the Loring, Andersen, and Fayer ${ }^{7}$ (LAF) solution to the exciton master equation as well as introducing several modifications to the LAF model.

In a previous paper ${ }^{8}$ we presented an experimental technique, and results, for studying triplet excitons in mixed naphthalene crystals at low temperatures $(1.8-16 \mathrm{~K})$. The guest species was naphthalene $\mathrm{C}_{10} \mathrm{H}_{8}$ while perdeuteronaphthalene $\mathrm{C}_{10} \mathrm{D}_{8}$ acted as the inert host species. We varied the guest concentration $C_{g}$ across the range of 0.20 to 1.0 mole fraction. Betamethylnaphthalene $B M N$ was also present in dilute amounts: $C_{s} / C_{g}=10^{-3}$ to $10^{-5}$. The BMN molecules acted as sensor, or trap, sites. We found that at

\footnotetext{
a) Supported by NSF Grant No. DMR 8303919.

b) Present address: Hughes Aircraft Co., 600/F245, P.O. Box 3310, Fullerton, California 92631.
}

$1.8 \mathrm{~K}$ the rate of transport $K^{R}$, within the guest quasilattice, exhibits a smooth functional dependence on guest concentration via the empirical relationship $K^{R} \propto C_{G}^{+6.5} . K^{R}$ is defined as $K^{R}=k C_{g} / C_{s}$, where $k$ is the rate constant for exciton trapping by the $\mathrm{BMN}$ sites.

In order to analyze the experimental results we will assume that the excitons can be described using a random hopping model, i.e., the excitons are localized entities which jump incoherently from one site to another. This random motion can be expressed using a master equation. A very different approach states that decreasing the guest concentration causes a quantum mechanical transition in the fundamental nature of the exciton. ${ }^{9-12}$ We will not consider the latter theory here since the transport data in Ref. 8 show a smooth functional dependence on $C_{g}$ across the entire concentration range of $C_{g}=0.20$ to 1.0 mole fraction. Assuming that excitons can be described as random walkers, $K^{R}$ can be interpreted as the effective rate at which an exciton samples new sites, and it should be proportional to the exciton diffusion coefficient. ${ }^{3}$

In Sec. II we describe the transport master equation and several approximate solutions to it which are found in the literature. Section III presents theoretical values for the superexchange matrix elements in naphthalene. Knowledge of these values is necessary in order to use the models presented in Sec. II. Section IV compares the consequent theoretical predictions for triplet exciton transport to the experimental results from Ref. 8 . We make several modifications to the master equation model of Loring, Andersen, and Fayer ${ }^{7}$ in Secs. V and VI and again compare the results to the experimental data. In Sec. VII we explain our results in terms of the transport dependence on the local environment. We also compare our triplet results to our previously described sing 
let exciton results. ${ }^{42}$ Section VIII summarizes our conclusions.

\section{TRANSPORT MASTER EQUATION}

The master equation for transport and trapping within a system of $N$ guest sites and $(M-N)$ trap sites is

$$
\frac{d p_{j}(t)}{d t}=-\sum_{k=1}^{N} p_{j} w_{j k}+\sum_{k=1}^{N} p_{k} w_{k j}-\sum_{k=N+1}^{M} p_{j} v_{j k},
$$

where $p_{j}(t)$ is the probability that guest site $j$ is occupied by an exciton at time $t . w_{j k}$ is the rate of exciton transfer from guest site $j$ to guest site $k . v_{j k}$ is the rate of irreversible transfer to trap site $k$. For most of this paper we will assume that we are in the high-temperature limit with respect to guest-guest transfer $w_{j k}=w_{k j}$ but not with respect to back transfer from trap sites.

This master equation is typically used to solve for configurationally averaged transport Green's functions, e.g., $G^{g}(\mathbf{r}, t)$, or their Fourier-Laplace transforms $\widehat{G}^{g}(\mathbf{k}, u) . \mathbf{k}$ is the Fourier variable for position while $u$ is the Laplace variable for time. $G^{g}(\mathbf{r}, t)$ gives the probability that an exciton resides on any guest molecule at location $\mathbf{r}$ and time $t$, given that the exciton was at the origin at time zero. The configurational average refers to solving for the Green's function for the case of a given guest and trap site distribution, e.g., $g^{g}(\mathbf{r}, t)$, and then finding the average functional value over all possible guest and trap site distributions in space.

$$
G^{g}(\mathbf{r}, t) \equiv\left\langle g^{g}(\mathbf{r}, t)\right\rangle=\Omega^{-M} \int_{\Omega} d \mathbf{r}_{1} \ldots \int_{\Omega} d \mathbf{r}_{N} \ldots \int_{\Omega} d \mathbf{r}_{M} g^{g}(\mathbf{r}, t),
$$

where $\mathbf{r}_{j}$ is the location of the guest or trap site $j . \Omega$ is the lattice volume, area, or length depending on the dimensionality of the system.

The Green's function approach is common to a number of solutions to the master equation problem. Huber ${ }^{15}$ uses these expressions to derive a $T$-matrix solution. A second type of solution is the coherent medium approximation. ${ }^{16-19}$ We will use a method which was initially proposed by Haan and $\mathrm{Zwanzig}^{20}$ in 1978 and subsequently improved upon by Gochanour, Andersen, and Fayer ${ }^{21}$ (GAF). The GAF model uses diagrammatic series to solve for the exciton transport. Concomitant with these series is the GAF requirement that the total exciton population probability remains conserved. Loring, Andersen, and Fayer ${ }^{7,43}$ (LAF-I) have extended the GAF model to include exciton trapping. The resultant equations that we will use for this paper are Eqs. (8), (9), and (10) in Ref. 43.

We will also make use of an earlier transport/trapping model suggested by Blumen and Silbey ${ }^{22}$ (BS). This model is less complete than the LAF-I model, but it is also much simpler to use. Rather than trying to explicitly calculate the long time behavior of the exciton distribution propagation, BS extrapolate from the short time behavior. They have solved for the trapping rate constant $k$ for a variety of conditions.

\section{DISTANCE DEPENDENCE OF NAPHTHALENE SUPEREXCHANGE INTERACTIONS}

The relevant question is how well do the LAF-I and BS solutions explain the experimental transport vs $C_{g}$ data presented in Ref. 8. In order to answer this question we must first know something about $w\left(\mathbf{r}_{i j}\right)$ for naphthalene triplets.

Naphthalene nearest neighbor molecules transfer triplet excitation via exchange interactions. ${ }^{23}$ Since this process requires the overlap of orbitals from the two sites, this interaction is extremely short ranged. Transfer between sites which are further apart than nearest or next-nearest neighbors is generally assumed to occur via superexchange. ${ }^{24-26}$ This process is similar to direct exchange except that it uses intervening host site orbitals to help propagate the electron exchange. If the nearest neighbor exchange matrix element is given by $\beta$ and the guest-host energy difference is $\Delta E$, then the superexchange matrix element, $J$ between two guest sites is $^{26}$

$$
J=\frac{L \beta^{m}}{\Delta E^{m-1}} \text { for } \beta<\Delta E
$$

$m$ is the number of nearest neighbor bonds belonging to the shortest route from one guest site to the other (passing through $m-1$ host sites). $L$ is the number of shortest distance paths between the two guest sites.

In order to calculate specific values of $J_{i j}$ we modified Eq. (3) slightly to allow for direct exchange between both nearest neighbor sites $\left[r_{n n}=0.5(a+b), \beta_{n n}=1.16 \mathrm{~cm}^{-1}\right]$ and next-nearest neighbors $\left(r_{n-n n}=b, \beta_{n-n n}=0.63\right.$ $\left.\mathrm{cm}^{-1}\right) .{ }^{27} \mathrm{We}$ also added the contribution of superexchange along the second shortest pathway to the contribution from the shortest pathway between two sites. The resultant matrix elements, for a large number of sites close to the origin, vs their corresponding distances from the origin are plotted in Fig. 1. The data yield the approximate two-dimensional isotropic relationship:

$$
J(r) \simeq A \exp \left[-4.3\left(\frac{r}{r_{n n}}-1\right)\right],
$$

where $r_{n n}$ is the nearest neighbor distance $(5.1 \AA)$. This expression is quite close to the rough superexchange estimate made by Blumen and Silbey. ${ }^{22}$ Instead of the value of 4.3, they predict a value of 4.5 using the general expression $-\ln (\beta / \Delta E)=4.5$.

If we assume that transport is incoherent then Fermi's Golden Rule should hold, i.e., $w_{i j} \propto J_{i j}^{2}$. This means that Eq. (4) gives a naphthalene triplet transfer rate which can be expressed using Eq. (5) with a value of $\epsilon=8.6$.

$$
w(r)=\tau_{n n}^{-1} \exp \left[-\epsilon\left(\frac{r}{r_{n n}}-1\right)\right] .
$$

$\tau_{n n}$ is the nearest neighbor transfer time. Several papers ${ }^{22.28}$ have treated the superexchange matrix element as being proportional to the matrix element rather than its square. This assumes that the transport is coherent. ${ }^{29}$ 


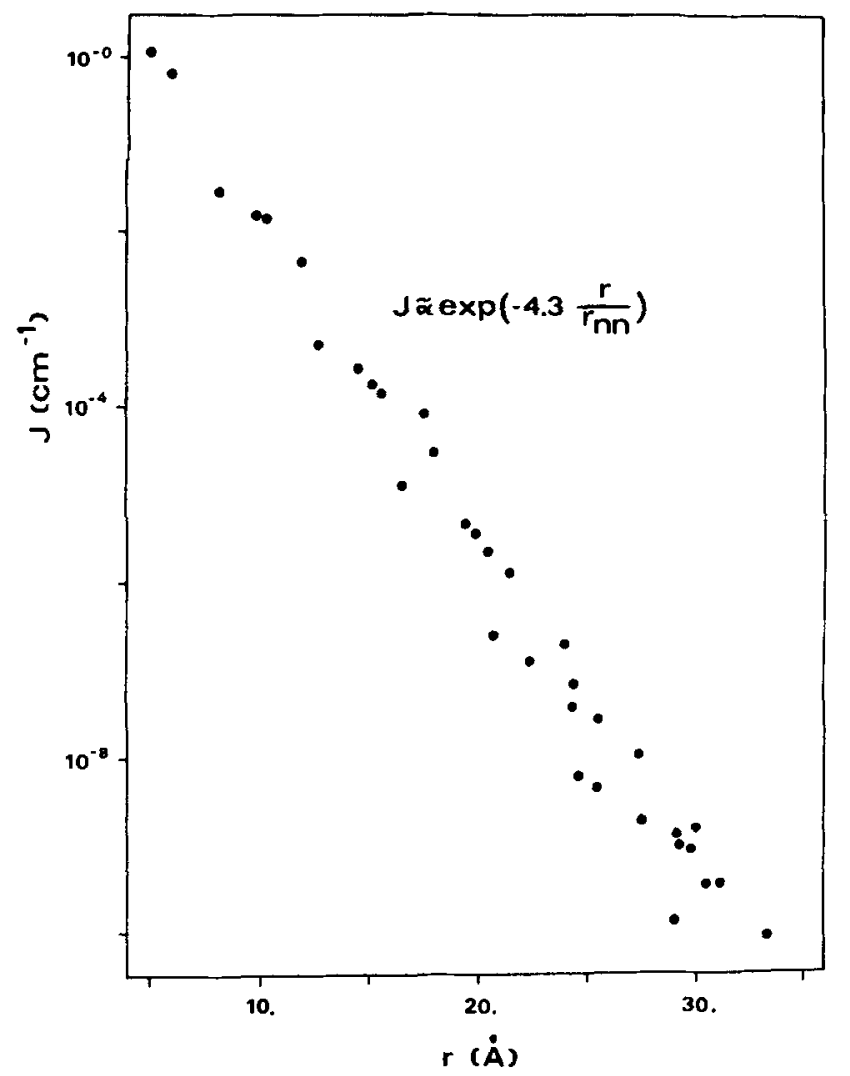

FIG. 1. Superexchange matrix elements for naphthalene. The matrix elements for transfer in the $a b$ crystal plane were calculated using Eq. (3). The data are based on direct exchange between nearest neighbors $[\beta(n n)=1.16$ $\left.\mathrm{cm}^{-1}\right]$ and between next-nearest neighbors $\left[\beta(n-n n)=0.6 \mathrm{~cm}^{-1}\right]$ (Ref. 27). The data also allow for contributions from both the shortest paths between two sites and the second shortest pathways. The data approximate the empirical relationship $J(r) \simeq A \exp \left(-4.3\left[\left(r / r_{n n}\right)-1\right]\right)$.

\section{COMPARISON OF EXPERIMENT TO STANDARD THEORIES}

Once one has found an expression for $w(r)$, one is able to make theoretical predictions using the LAF-I or BS models and compare these predictions to our experimental results. The hope is that the models will provide us with some insight into the physical process of triplet exciton transport in naphthalene crystals.

Using Eq. (5) we fit the LAF-I and BS theories to the data in Ref. 8. Unless otherwise specified, we have assumed that transport is incoherent $(\epsilon=8.6)$ and that the rate of guest-to-supertrap transfer is the same as guest-to-guest transfer for equal separation distances. This leaves only one adjustable parameter in the theories, i.e., the nearest neighbor transfer time $\tau_{n n}$. Results from the LAF-I model were calculated by solving the two-body equations using numerical integration. In order to extract the trapping rate constant $k$ from the LAF-I model we first solved for the function $\hat{G}^{g}(0, u)$ which gives the total population of excitons lying within the guest system of sites. We then used the Stehfest ${ }^{30}$ inverse Laplace transform method to obtain $\widehat{G}^{g}(0, t)$. Figure 2 (bottom abscissa) shows resultant $\hat{G}^{g}(0, t)$ values for $C_{8} \simeq 1.0$ and $C_{s}=10^{-3}$ as a function of the reduced time $t / \tau_{n n}$. (The top abscissa and the interpretation of the reduced time will be discussed in the next section.)

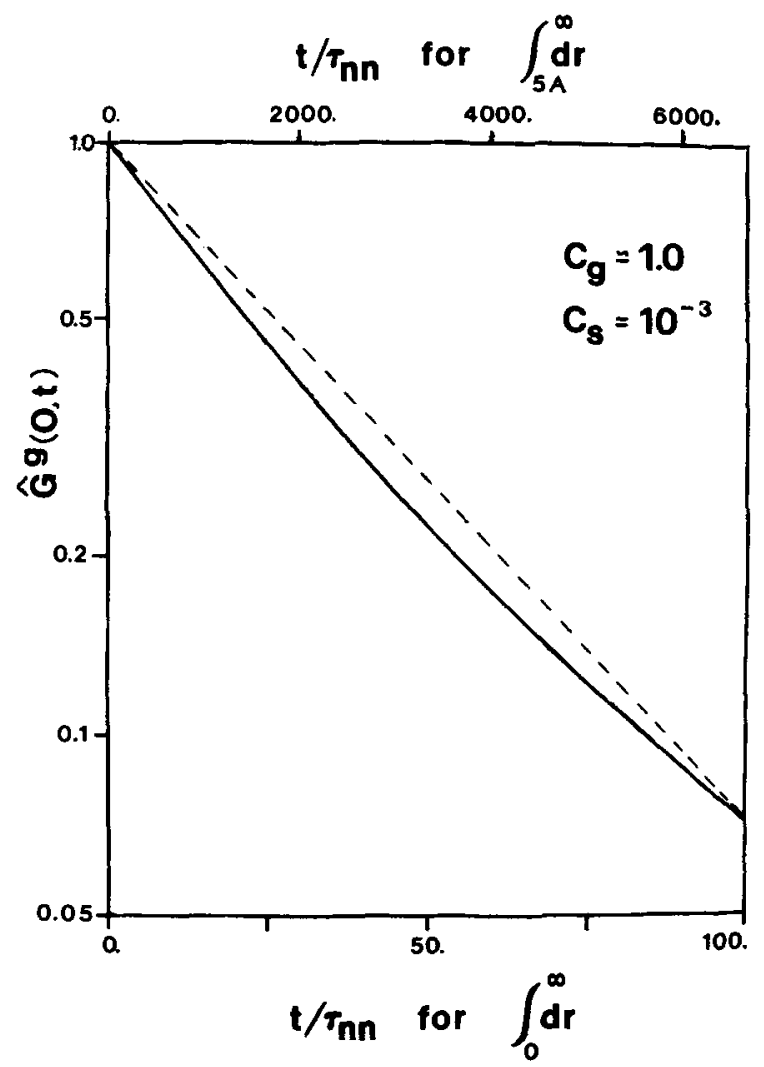

FIG. 2. LAF-I guest population as a function of time and as a function of lower integration bound. The solid line was calculated using the LAF-I model with $\epsilon=8.6$ and by using a lower integration bound of 0 and of $5 \AA$ (top scale). The guest population is plotted as a function of the reduced time for the two different lower integration bounds. The curves obtained for the two different lower bounds were virtually indistinguishable except for the magnitude of the time axes. The dashed line represents an exponential decay function.

While Fig. 2 shows that $\widehat{G}^{g}(0, t)$ is not an exponential function, the deviation from exponentiality is only on the order of the-noise seen in our experimental time-resolved spectra. This deviation from exponential behavior was expected $^{31-33}$ and did not change significantly when we subsequently modified the LAF-I model. Since our goal was to interpret our experimental results, we used our laboratory data analysis procedure to quantify our theoretical calculations. To do this we calculated $\widehat{G}^{g}(0, t)$ over the time range corresponding to $\widehat{G}^{g}(0, t=0)$ to $\hat{G}^{g}(0, t)=1 / e^{2}$. We then used least squares linear regression to fit the calculated points to an approximate exponential decay function with a trapping rate constant of $k$.

Figure 3 compares the experimental data to the results which are obtained from the LAF-I and BS models. The best fit with the data is obtained when using the LAF-I model with $\epsilon=8.6$ and with $\tau_{n n}=10 \mathrm{~ns}$. In all cases, the effect of changing $\tau_{n n}$ was to cause the theoretical curves to be uniformly translated in the vertical direction on a $\log [k]$ vs $\log \left[C_{g}\right]$ plot. The nearest neighbor naphthalene triplet transfer time is usually thought to be on the order of 10 to $100 \mathrm{ps}^{34}$ rather than $10 \mathrm{~ns}$. While it is true that there is some ambiguity in how this transfer time is defined in various papers, it is not likely that either this ambiguity or the differences in temperatures for our experiments and those of the von Burg $e t$ 


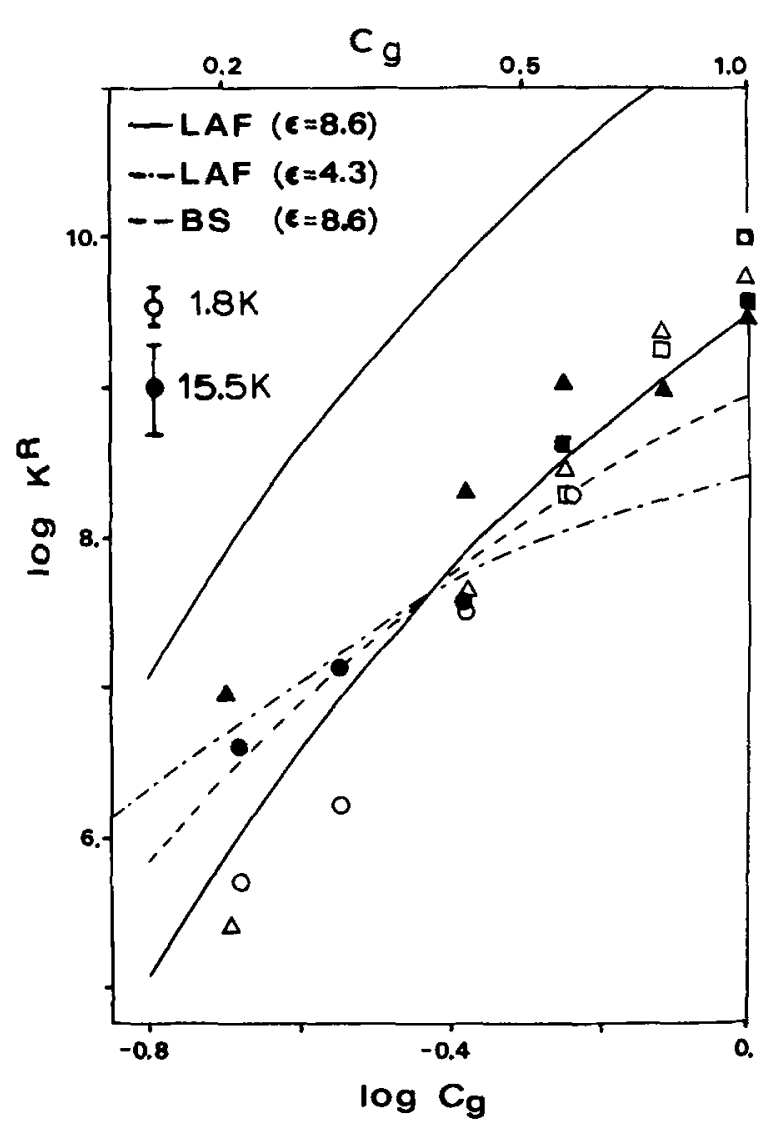

FIG. 3. Comparison of experimental data with standard theories. Experimental: The triangles are the data for $C_{s} / C_{g} \simeq 1 \times 10^{-3}$; the circles are for $C_{s} / C_{g} \simeq 2 \times 10^{-4}$; and the squares are for $C_{s} / C_{8} \simeq 4 \times 10^{-5}$ (from Ref. 8). The hollow data points are for $1.8 \mathrm{~K}$ while the solid data points were obtained at $15.5 \mathrm{~K}$. The two sets of data were taken on different days in different cryostats. Theoretical: The solid lines are for the two-body LAF-I model and using the incoherent value of $\epsilon=8.6$ for Eq. (5); the dot-dash line is the two-body LAF-I result using the coherent value of $\epsilon=4.3$; and the dashed line is the BS result with $\epsilon=8.6$. There are two LAF-I results based on $\epsilon=8.6$. The top curve assumes that $\tau(n n)=100 \mathrm{ps}$. The lower curve assumes that $\tau(n n)=10$ ns. The two $\epsilon=8.6$ LAF-I curves are identical except for a uniform vertical translation. The BS result is shown for $\tau(n n)=56$ ns. The $\epsilon=4.3 \mathrm{LAF}-\mathrm{I}$ result assumes that $\tau(n n)=18 \mathrm{~ns}$. With the exception of the $\tau(n n)=100 \mathrm{ps}$ LAF-I curve, the $T(n n)$ values were chosen to give the closest agreement with the experimental data. The best fit with the data is provided by the LAF-I model with $\epsilon=8.6$ and $\tau(n n)=10 \mathrm{~ns}$.

al. experiments ${ }^{34}$ will account for the two orders of magnitude discrepancy in hopping time. Similarly, it is not likely that the trapping efficiency $\eta_{t}$ [Eq. (7)], deviates by a factor of 100 from a value of $\eta_{t}=$ unity. ${ }^{35}$ Figure 3 also shows the result that is obtained when the coherent value of $\epsilon=4.3$ is used with the LAF equations. This result should not be taken too seriously, however, since we are applying a coherent parameter to what is an incoherent transport model.

Figure 3 plots experimental data for both 15.5 and 1.8 $K$. For a given crystal, the two data were obtained on different days and in different cryostats. Raising the temperature increases the quality of the agreement between theory and experiment slightly. This is not surprising since LAF solve the master equation for the high temperature limit.

\section{COMPARISON OF EXPERIMENT TO MODIFIED THEORIES}

A number of assumptions were made in deriving the equations used in Fig. 3. We have made several modifica- tions to the original LAF-I and BS theories in order to test these assumptions. This section will discuss the results due to changing the limits of integration used in the continuum model as well as varying the trapping efficiency. The succeeding section will discuss the result of changing from a continuum to an underlying lattice model.

One major assumption in both the BS and LAF-I models is the definition of the configurational average in Eq. (2). This equation says that we are to find the average of the transport function as we integrate the position vector of the $k$ th guest site across the entire space. Since by definition there is already one guest site at the origin, it is more realistic physically to forbid the $k$ th guest site from occupying a position less than the crystallographic nearest neighbor distance from the origin ${ }^{20}$

$$
\int_{0}^{\infty} d r_{k} F\left(r_{k}\right) \rightarrow \int_{r_{n n}}^{\infty} d r_{k} F\left(r_{k}\right)
$$

Changing the lower bound of the integration from 0 to $5 \AA$ provides a significant improvement in the best fit value of $\tau_{n n}$ relative to an expected value of $\tau_{n n} \simeq 100$ ps (Fig. 4, dashed curves). The problem with using the origin as the lower bound for the integration is that transport becomes too efficient owing to the rapid transfer between sites which are

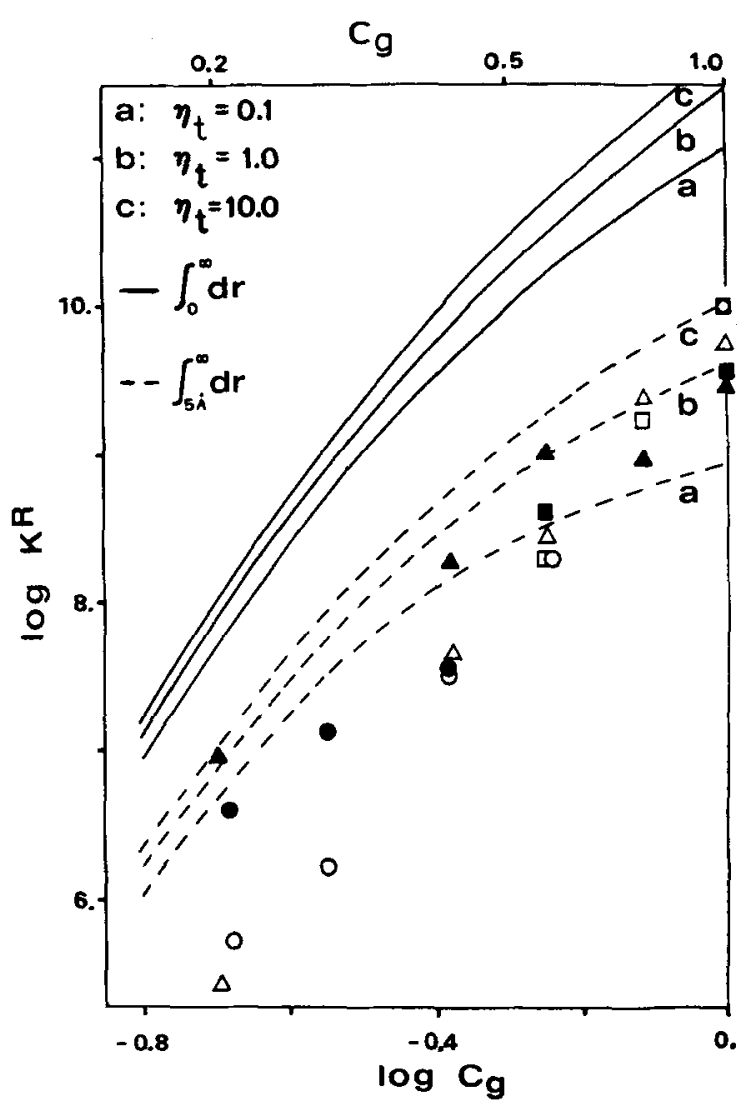

FIG. 4. Influence of the trapping efficiency on transport. Experimental: see Fig. 3. Theoretical: The solid and dashed lines refer to using a lower integration bound of 0 and $5 \AA$, respectively, for the modified LAF-I two-body equations. These integration bounds were used with three different trapping efficiencies $\eta_{t}$, as defined by Eq. (7). All of the curves are based on $\epsilon=8.6$ and $\tau_{n n}=100 \mathrm{ps}$. 
less than $5 \AA$ apart. [Using Eq. (5) with $\epsilon=8.6$ and $r_{n n}=5.1$ $\AA$ the superexchange rate constant at the origin is $w(r=0)=5431 w\left(r=r_{n n}\right)$. For multipolar transfer, $w(r)$ increases to a singularity as $r$ is decreased to 0 .] In order to compensate for the increased transport efficiency, one had to slow the overall process via the $\tau_{n n}$ scaling parameter. The introduction of a cutoff distance is neither trivial, nor does it introduce an adjustable parameter. The cutoff is similar to that which has proven invaluable in the Debye theory of heat capacity, and the nearest neighbor distance is a well-defined crystallographic quantity.

Another way of demonstrating the dependence on the lower bound of the configurational average integral is to look at the time decay of the calculated guest population, $\hat{G}^{8}(0, t)$ in Fig. 2. The bottom abscissa is the reduced time scale for the case of integrating $d \mathbf{r}_{k}$ from the origin and the top abscissa is $t / \tau_{n n}$ for the case of beginning the integration at $5 \AA$. With the exception of the difference in the magnitude of the two reduced time axes, the curves calculated for a lower integration bound of 0 and of $5 \AA$ are virtually indistinguishable, within the errors introduced by the inverse Laplace transform method. If we interpret $t / \tau_{n n}$ as being the approximate number of steps in a random walk, since $\tau_{n n}$ is the naphthalene nearest neighbor transfer time, then the lowerbound-equal-to-zero data are such that 84 steps are needed for the guest exciton population to decrease to 0.1. (In actuality, $\tau_{n n}$ has no physical meaning when the continuum extends to the origin other than as the transfer time for a randomly chosen distance.) This value is unrealistically low since $C_{s}=10^{-3}$ mole fraction implies that an exciton must visit an average of 1000 sites before it will find a trap site. Furthermore, due to site revisitation a random walker will require more than 1000 steps to visit 1000 sites. When the integration begins at $5 \AA$, then the data in Fig. 2 imply that 5630 steps are required to find a trap site. Our comparison of the $K^{R}$ vs $C_{g}$ curves for the two lower integration bounds (Fig. 4) was based on our experimental data and on our expectations of what $\tau_{n n}$ should be for naphthalene. The analysis of $\widehat{G}^{x}(0, t)$ vs $t$ (Fig. 2), on the other hand, is based entirely on theoretical considerations. The two types of analysis are consistent with one another relative to the inadvisability of using the origin as a lower integration bound when describing transport on a lattice.

A second variation which we made on the preceding results was to remove the restriction that the guest-to-guest and guest-to-trap transfer rates are the same for equal separation distances. This is not a modification to the LAF-I model since it was only in our implementation of their equations that we said that the two would be kept equal. The multiplicative factor which relates the two transfer rates is the trapping efficiency $\boldsymbol{\eta}_{t}$.

$$
\tau_{n n}(g \rightarrow s)=\eta_{t} \times \tau_{n n}(g \rightarrow g) .
$$

Figure 4 compares the LAF-I and our modified LAF-I twobody continuum results for three different trapping efficienies, $\eta_{t}=0.1,1.0$, and 10.0. The trapping efficiency is likely to be on the order of 2 to $4 .{ }^{42,56}$ As Fig. 4 shows, changing the trapping efficiency by a factor of 10 does not have a large effect on the calculated transport rate.

\section{CONTINUUM VERSUS LATTICE}

In the two preceding sections we were able to obtain a reasonable agreement between our experimental data and our modified LAF-I solution to the master equation. Unfortunately, these sections also demonstrated that it is difficult to obtain precise transport data by comparing theory and experiment, due to the number of variables which are present in the experimental system and the number of assumptions that are made in the theoretical calculations.

Until now, we have ignored the most severe assumption in the LAF-I and BS models, i.e., the question of transport in a continuum rather than on a lattice. The definition of the configurational average in Eq. (2) assumed that each of the guest sites can lie anywhere in space, except as limited by the dimensionality of the system. In the crystal, the positions of the naphthalene molecules are restricted to residing on an underlying ordered lattice. This means that we should replace an integration over all space with a summation over all lattice points:

$$
\int_{\Omega} d r F(r) \rightarrow \Omega \sum_{m} F\left(r_{m}\right)
$$

where $m$ is a lattice point index and $\Omega$ is the lattice volume, area, or length, depending on the dimensionality of the system.

The problem with inserting this transformation directly into the BS and LAF-I models is that neither model excludes two molecules from occupying the same point on a discrete lattice. This is not a problem when dealing with infinitesimal integration points. Blumen and $\mathrm{Manz}^{36}(\mathrm{BM})$ have introduced lattice equations which can be inserted into the BS model. Modifying the LAF-I equations to give a lattice rather than continuum result is more difficult than modifying the BS model. Loring, Andersen, and Fayer ${ }^{37,38}$ (LAF-II) have extended the LAF-II model to account for transport on a lattice. Unfortunately, the GAF model solves for the transport problem, not for the LAF-I trapping/transport problem. While it is possible to relate the two theoretically, in practice, the translation between the two is not simple. As an alternative, we chose to ignore the double occupancy problem and replace the LAF-I integrations with summations. The double occupancy problem was partially compensated for when subsequently normalizing the total probability with Eq. (8) in Ref. 43. At the end of this section we will demonstate that we get similar results to the lattice calculations by Loring et al..$^{37,38}$

Figure 5 compares the BS and the modified LAF-I lattice results to the experimental data. [We used the individual matrix elements shown in Fig. 1 rather than using the isotropic equation, Eq. (5)]. Both models give results which are dominated by a plateau in the transport rate above $C_{g} \simeq 0.40$ mole fraction. Because of the similarity in results from the two theories, it is highly unlikely that the inflection point in the modified LAF-I result is due merely to the double occupation problem. Furthermore, using the LAF-I three-body equations ${ }^{7,39}$ (with $r_{2} \neq r_{3}$ ) rather than the two-body equations (used elsewhere in this paper), made the problem worse instead of better. The existence of a transport plateau for $C_{g}>0.40$ is likely due to the large difference in transfer rates 


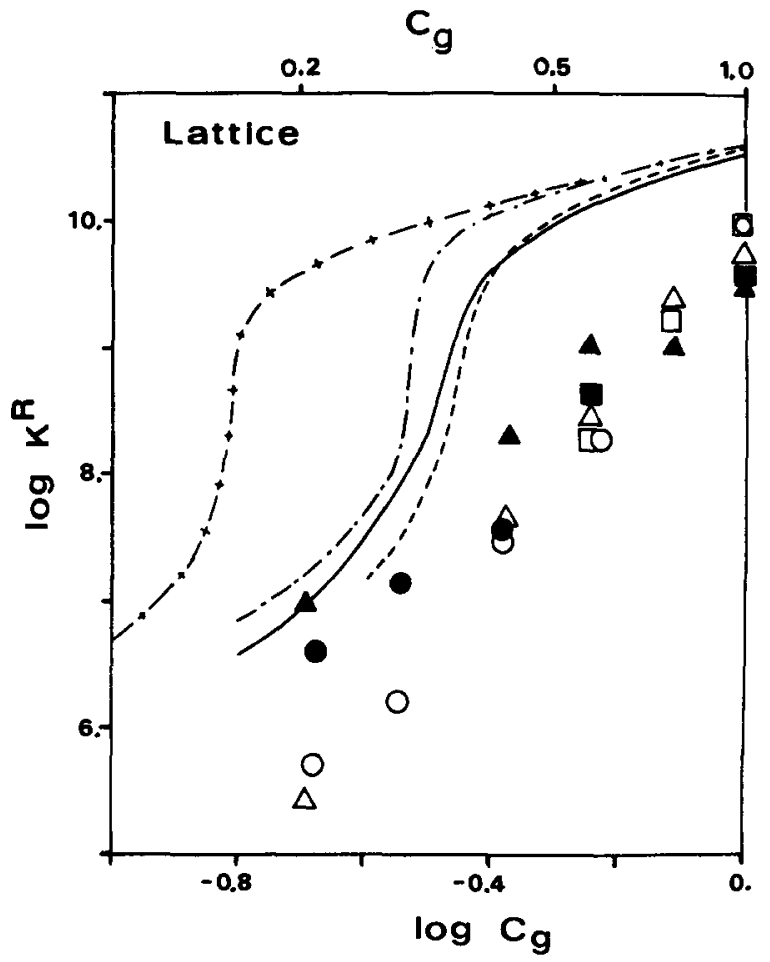

FIG. 5. Transport on a lattice. Experimental: $1.8 \mathrm{~K}$; see Fig. 3. Theoretical: The solid line is the modified LAF-I two-body lattice result; the dashed line is the modified LAF-I three-body lattice result; the dot-dash line is the BS lattice result for the case where back transfer to the origin in neglected; and the cross-dash line is the BS lattice result allowing for back transfer. All of the curves are based on the square of the superexchange matrix elements shown in Fig. 2 and with the lattice summations and products carried out to the 48th-nearest neighbors. $\tau_{n n}$ was assumed to be $100 \mathrm{ps.}$ for nearest, next-nearest, and third-nearest neighbors (cf. Fig. 1).

The lattice calculations in Fig. 5 were made by including superexchange transfer out to the 48th-nearest neighbors in naphthalene. In order to probe our lattice results further, we did similar calculations for the case where transfer is limited to nearby sites. Figure 6 shows the results for $n=1$, 2,3 , and 48, where $n$ is the number of neighbor lattice shells used in the transport calculations. We choose $n=48$ in order to simulate the case where there are nonzero transfer rates to "all" of the guest sites on an infinite lattice. Figure 6 plots these $K^{R}$ vs $C_{g}$ theoretical data on both a $\log$ plot and a linear plot. Figure 6 also includes a superexchange LAF-I continuum result for the sake of comparison.

There are several interesting features in Fig. 6. The linear plot demonstrates that the plateau behavior seen in Fig. 5 translates into a linear concentration dependence for $C_{g}>0.40: K^{R} \propto C_{g}^{+1.0}$ as opposed to our experimental $K^{R} \propto C_{g}^{+6.5}$. A second feature is the fact that there are critical concentrations below which $K^{R}$ is undefined. These critical concentrations are isomorphic with percolation critical concentrations. For $n=1,2$, and 3, the modified LAF-I critical concentrations for the naphthalene lattice are $C_{c}=0.499,0.333$, and 0.250 , respectively. For a triangular lattice with $n=1$ or 2 the critical concentrations are $C_{c}=0.333$ and 0.167 . For a square lattice with $n=1$ or 2 , $C_{c}=0.499$ and 0.250 . To a very good approximation all of these critical concentrations are consistent with an empirical relationship which depends only on the number of guest sites used in the lattice summation $m: C_{c}=2 / m$. The $C_{c}$ vs $m$
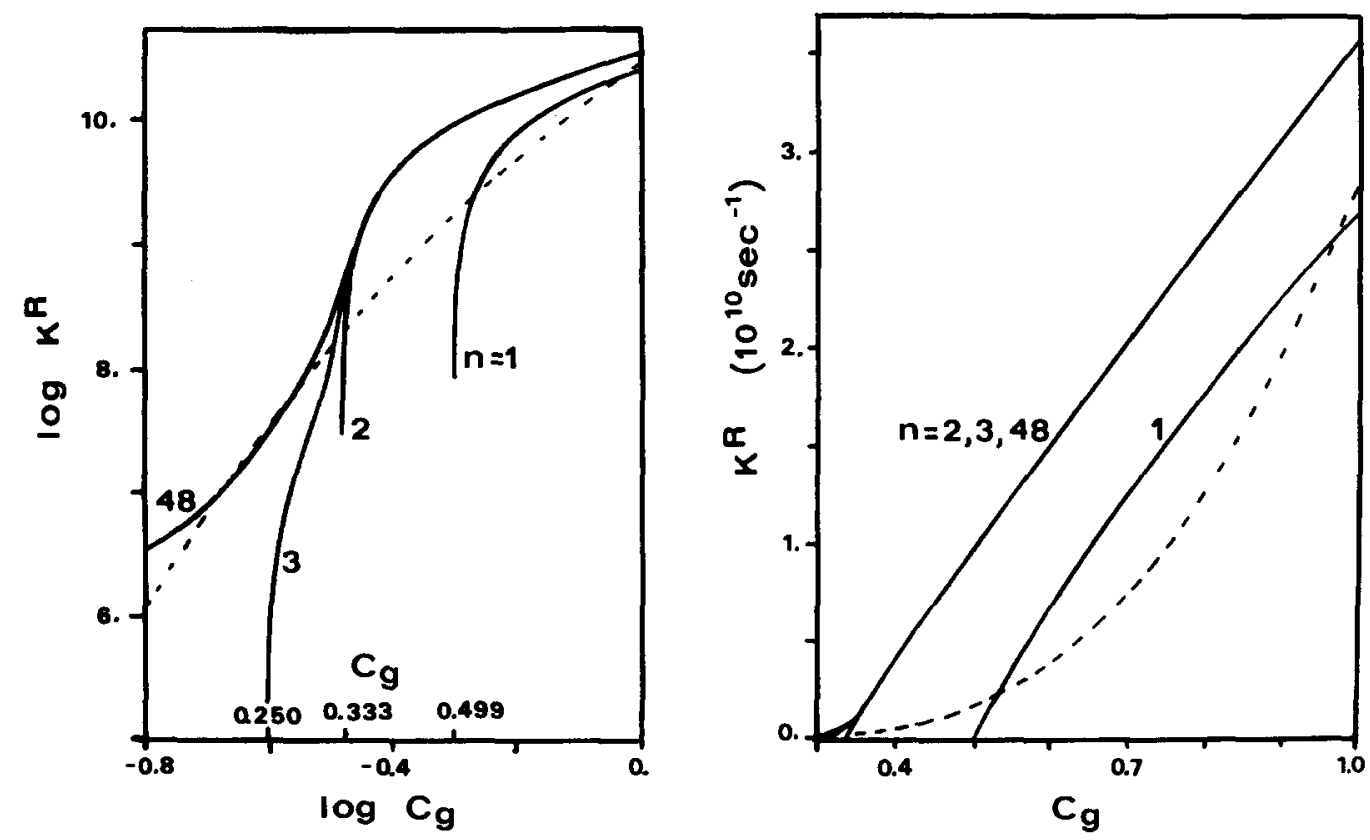

FIG. 6. Critical concentrations due to lattice. The solid lines are modified LAF-I two-body lattice results based on $\pi(n n)=100 \mathrm{ps}$ and on the square of the matrix elements shown in Fig. 2. The various solid lines were obtained using different values of $n$, where $n$ is the number of neighbor lattice shells used in the calculations. For example, $n=2$ means that only nearest and next-nearest neighbor transfer interactions were used. $n=48$ was chosen in order to simulate the case where there are nonzero transfer rates to all of the guest sites on an infinite lattice. If we had continued the calculations to lower guest concentrations, however, presumably the $n=48$ curve would also have a critical concentration. While the $n=2,3$ and 48 curves are barely distinguishable from one another on the linear plot, they are clearly separable on the log plot. The dashed line is the LAF-I two-body continuum result based on $\epsilon=8.6$ and $\tau(n n)=1.0 \mathrm{~ns}$. The results are shown on both a log plot and a linear plot. 
equation also agrees with the well known result ${ }^{40}$ that the long time limit for transport in a one-dimensional system which is limited to nearest neighbor interactions is nonzero only for the case of a pure crystal $C_{g}=1.0$. An interesting feature of the $C_{c}$ equation is that it does not depend on the relative transfer rates to the various $m$ sites. This demonstrates that the critical concentration is a function of the lattice statics rather than a dynamic property of the system.

Korzeniewski, Friesner, and Silbey ${ }^{18}$ (KFS) have done lattice calculations using the coherent medium approximation $^{17}$ and Loring, Andersen, and Fayer ${ }^{37,38}$ have done numerical calculations using their LAF-II model. (Korzeniewski and Calef $^{19}$ have extended the coherent medium model, and done calculations, to include some cluster effects which are not included in the KFS model. Their results are limited, however, to the case of a random walker limited to nearest neighbor hops.) Both KFS and LAF-II observed a linear transport dependence on concentration similar to our results. They calculate the diffusion coefficient rather than $K^{R}$; but, as stated in Sec. I, the two should be proportional. KFS and LAF also observed the critical concentration phenomenon (cf. Fig. 6), with $C_{c}$ values that are consistent with our values. The qualitative similarity between our results and the results based on the more complete KFS and LAF-II models, as well as our BM/BS results, seems to indicate that the discrepancy between our lattice calculations and our experimental data is not merely due to the non-self-consistency of our model.

We are left, therefore, with the inconsistency that our experimental data, which are obtained from naphthalene in a crystalline lattice, are more consistent with continuum than with lattice transport models. Our explanation for this inconsistency is that even though the guest sites do not lie in a continuum, the transfer rate constants do still lie in a continuum. We can expand on this conclusion by pointing out that the symmetry of the lattice only enters most lattice models by limiting the incoherent pairwise transfer rate constants to a set of discrete values. If the transfer rate constants are not limited to a discrete set, then the LAF-I continuum model should be valid. There are several possible explanations for there being a spread in the transfer rate constants for two sites separated by a given distance. One is that the transfer rate depends not only on the position of the two sites relative to one another, but also on the local environment of the sites. (This will be discussed further in the next section.) A second explanation is the possibility that the excitons are not always strictly localized on one guest site.

\section{INFLUENCE OF LOCAL ENVIRONMENT ON TRANSPORT}

Models such as those presented by Loring, Andersen, and Fayer and by Blumen and Silbey consider only the first order effect of having a randomly mixed lattice: decreasing the guest concentration increases the average guest site separation distance. Even at this level of approximation solving the master equation is formidable due to the problem of correctly averaging all of the possible guest and trap site distributions. A higher order effect which the LAF and BS models do not take into consideration is the influence of the

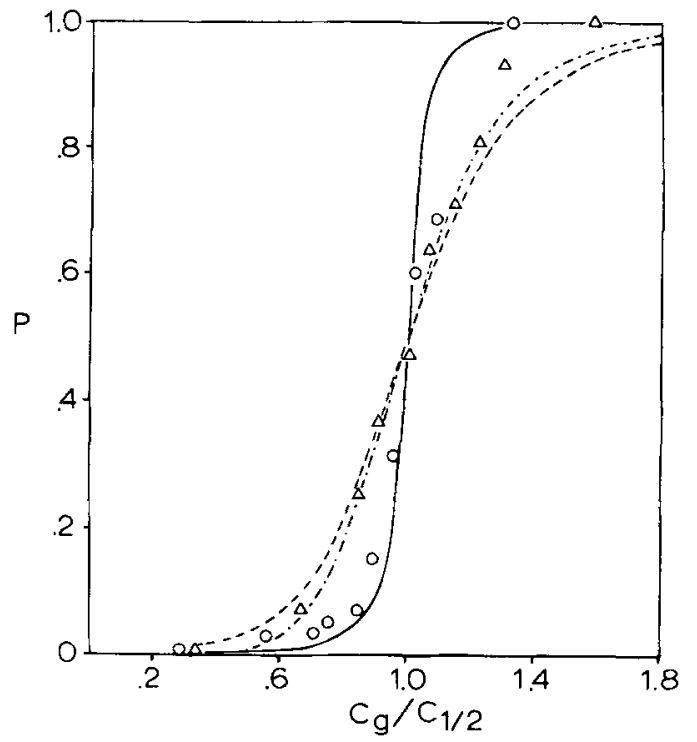

FIG. 7. Trapping probability vs reduced concentration. $C(1 / 2)$ is defined as the concentration corresponding to $P=0.5$. Experimental: Circles are 1.8 $\mathrm{K}$ data; Triangles are $4.2 \mathrm{~K}$ data. Theoretical: The solid and dashed lines correspond to percolation and the Loring and Fayer calculation, respectively. The dot-dashed line was calculated using the Blumen and Silbey model. The Blumen and Silbey and the Loring and Fayer curves assume octupoleoctupole transfer within a two-dimensional continuum. The experimental data and the percolation and Blumen and Silbey curves were calculated using $C_{s} / C_{g}=0.001$. The Loring and Fayer curve kept $C_{s}$ constant at $C_{s}=0.001$. Changing the Loring and Fayer curve to account for a constant $C_{s} / C_{g}$ ratio would only add a small correction. As a comparison, if the Blumen and Silbey equations were calculated using $C_{s}=0.001$ then the result would lie halfway between the dot-dashed and the dashed lines for $C_{g} / C(1 / 2)<1.0$. For $C_{g} / C(1 / 2)>1.0$, the result is essentially identical to the Loring and Fayer curve. The percolation curve is based on a square lattice with transfer limited to nearest neighbor jumps. See Ref. 42 for a full discussion of how the experimental and theoretical results were obtained.

surrounding guest and host sites on the individual pairwise transfer rates.

Although the local environment has only a small effect on our 1.8-15.5 K triplet results, i.e., requiring the use of a continuum rather than lattice model, it has a large effect on the naphthalene singlet transport for the same temperature and concentration ranges. This can be seen in Fig. 7, which was taken from Ref. 42 . The singlet data are given in terms of the trapping probability $P$. This is the probability that an exciton will find a trap site within the exciton lifetime $\tau_{g} . P$ can be related to the kinetic trapping rate constant, used elsewhere in this paper, via

$$
P=\frac{k}{k+\tau_{g}^{-1}}
$$

$P$ can be found experimentally ${ }^{42}$ by measuring the relative guest and trap fluorescence intensities. Also, Loring and Fayer ${ }^{43}$ show how $P$ can be calculated using the LAF-I model.

Reference $\mathbf{4 2}$ gives a full discussion of the experimental data in Fig. 7 with respect to the LAF-I model, the BS model and percolation. What is important for the current discussion is that when the temperature was lowered to $1.8 \mathrm{~K}$, the singlet transport showed a much stronger dependence on guest site concentration than could be explained by the standard LAF-I model. None of the modifications described previously, i.e., changing the trapping efficiency, changing the 
integration bound or doing a lattice calculation, gave a substantially better fit with the experimental results. The $1.8 \mathrm{~K}$ singlet data did give a reasonable fit with the predictions made by a percolation model ${ }^{51-53}$ which limits excitons to moving within the boundaries of the guest site clusters.

Our explanation for the singlet data is that as we lower the temperature and/or guest concentration, the rate of transfer from site $i$ to site $j$ is no longer the same as that in the reverse direction, i.e., $w_{i j} \neq w_{j i}$. This happens when the thermal energy becomes small relative to the energy mismatches between guest sites. In mixed crystals, the energy mismatches are primarily the result of the random distribution of finite guest cluster sizes. In general, the more guest sites which belong to a cluster, the lower is the minimum exciton energy state of that cluster. In a $C_{g}=0.30$ mole fraction crystal, the weighted average of singlet energy mismatches between clusters is $\delta \simeq 5 \mathrm{~cm}^{-1} .^{28}$ At low temperature (i.e., singlet excitons at $1.8 \mathrm{~K}$ ) the excitons will rapidly cascade to the lowest lying clusters where they are subsequently confined by the cluster boundaries.

In addition to detailed balancing, the energy mismatches which arise from the guest site clusterization affect transfer through quantum mechanical considerations due to exciton-phonon damping. Parson and Kopelman ${ }^{48}$ have shown that even in the high temperature limit, transfer from a dimer to a monomer, or vice versa, is slowed down as the energy splitting of the dimer increases. They derived this energy mismatch effect by applying the Haken and Strobl ${ }^{49}$ stochastic model for exciton dynamics. Like the detailed balance effect, this quantum mechanical attenuation of the transfer rate between clusters should play an increasingly important role in the transport as the guest concentration is reduced, i.e., as the breaking up into clusters and the average energy mismatch are increased.

As we have seen, triplet excitons under similar conditions $\left(T=1.8 \mathrm{~K}, C_{g}=0.20-0.70\right.$ mole fraction) do not show signs of cluster confinement. The smaller bandwidth of the triplet $\left(10 \mathrm{~cm}^{-1}\right.$ vs $150 \mathrm{~cm}^{-1}$ for the singlet) means that one must go to a much lower temperature before the thermal energy decreases enough to be on the order of $\delta$. The alternative is to reduce the guest concentration. As the average cluster size decreases, the average energy mismatch for the clusters increases. This cluster size dependence on concentration may explain why we did not observe triplet exciton cluster confinement for $C_{g}>0.20$ crystals whereas previous work on $C_{g}<0.20$ crystals did show a sizeable deviation from diffusion, or homogeneous kinetics. ${ }^{44-47}$ The $C_{g}$ dependence is further enhanced since decreasing the guest concentration also slows down the overall transport rate until it is on the order of the natural lifetime of the exciton.

\section{SUMMARY}

In summary, we have analyzed our data with three versions of the LAF-I theory, as well as with the simpler Blumen-Silbey and Blumen-Manz theories. We found that the original continuum version of the LAF-I theory gave a fairly good description of our data for a hopping rate considerably smaller than the generally accepted value. We conjectured that this problem was due to a physically unrealistic contri- bution from transfer events involving molecules separated by less than a molecular radius. Following a procedure common in lattice-statistical problems ${ }^{54}$ previously applied in this context by Haan and Zwansig ${ }^{20}$ we inserted a short distance cutoff on the transition rate. We found that a cutoff corresponding to the known intermolecular spacing allowed the data to be fitted with a transfer rate reasonably close to the values suggested by independent experimental measurements. ${ }^{34}$ Emboldened by this success, we tried to get even closer agreement by replacing the continuum by a lattice. Unlike our modified cutoff of the LAF-I theory, our lattice calculations suffer from internal inconsistencies: the averaging procedure accounts for excluded volume effects but the quantities averaged do not. However, we do not think that this inconsistency accounts for the qualitative changes in the theoretical prediction, since the same effects arise in the Blumen-Manz calculation. We believe, rather, that the shape of the theoretical $K^{R}$ vs $C$ curve arises from the discontinuous distribution of hopping rates in the system; it is a highly rounded and smoothed analog of the percolation edge which occurs when the interaction range is not finite.

Naturally, we cannot ascribe too much quantitative significance to the lattice results, and we certainly do not expect a non-self-consistent theory to be able to interpolate through a genuine percolation edge. Very recently, Long et al. have developed the trapping analog of the LAF-II self-consistent lattice transport theorys5; the application of this theory to our data will determine whether the inflection in the $K^{R}$ vs $C$ is an artifact. Until then, our conclusions remain tentative. In the meantime, we propose that the difference between our experimental results and our theoretical lattice results is largely due to the distribution of site energies resulting from the random clusterization of guest sites.

Exciton transport in isotopically mixed molecular crystals is a very challenging problem. Invariably any theoretical model which attempts to solve for the exciton transport parameters in such a system must include a number of assumptions to make the model tractable. While these assumptions may be oversimplified, modifying the assumptions and comparing the calculated results to experimental data can be a valuable tool for understanding excitons in disordered media.

\section{ACKNOWLEDGMENTS}

We would like to thank Robert Parson for many fruitful theoretical discussions and help with this manuscript. We also thank R. F. Loring and M. D. Fayer for sending us a preprint of their work as well as for several private communications.

${ }^{1}$ R. W. Munn and R. Silbey, Mol. Cryst. Liq. Cryst. 57, 131 (1980).

${ }^{2}$ G. Zumofen and A. Blumen, J. Chem. Phys. 76, 3713 (1982).

${ }^{3}$ V. M. Kenkre and D. Schmid, Chem. Phys. Lett. 94, 603 (1983).

${ }^{4}$ Th. Förster, Ann. Phys. 2, 55 (1948) (English translation by R. S. Knox, University of Rochester, New York, 1974)

${ }^{5}$ D. L. Dexter, J. Chem. Phys. 21, 836 (1953).

${ }^{6}$ R. A. Auerbach, G. W. Robinson, and R. W. Zwanzig, J. Chem. Phys. 72, $3528(1980)$.

${ }^{7}$ R. F. Loring, H. C. Andersen, and M. D. Fayer, J. Chem. Phys. 76, 2015

(1982); 77, 1079 (1982). 
${ }^{8}$ S. T. Gentry and R. Kopelman 81, 3014 (1984).

'J. Klafter and J. Jortner, Chem. Phys. Lett. 49, 410 (1977).

${ }^{10} \mathrm{P}$. W. Andersen, Phys. Rev. 109, 1492 (1958).

${ }^{11}$ N. F. Mott, Rev. Mod. Phys. 50, 203 (1978).

${ }^{12}$ C. M. Soukoulis, J. Klafter, and E. N. Economou, Solid State Commun. 44, $833(1982)$

${ }^{13}$ K. Godzik and J. Jortner, J. Chem. Phys. 72, 4471 (1980).

${ }^{14}$ D. Bedeaux, K. Lakatos-Lindenberg, and K. E. Shuler, J. Math. Phys. 12, 2116 (1971).

${ }^{15}$ D. L. Huber, Phys. Rev. B 20, 2307 (1979).

${ }^{16}$ I. Webman, Phys. Rev. Lett. 47, 1496 (1981).

${ }^{17} \mathrm{M}$. Lax and T. Odagaki, in Lecture Notes in Physics 154, edited by $\mathbf{R}$ Bumridge, S. Childress, and G. Papanicolaou (Springer, Berlin, 1983).

${ }^{18} \mathrm{G}$. Korzeniewski, R. Friesner, and R. Silbey, J. Stat. Phys. 31, 451 (1983).

${ }^{19}$ G. Korzeniewski and D. F. Calef, J. Phys. Chem. 16, 4599 (1983).

${ }^{20}$ S. W. Haan and R. Zwanzig, J. Chem. Phys. 68, 1879 (1978).

${ }^{21}$ C. R. Gochanour, H. C. Andersen, and M. D. Fayer, J. Chem. Phys. 70 4254 (1979).

${ }^{22}$ A. Blumen and R. Silbey, J. Chem. Phys. 70, 3707 (1979).

${ }^{23}$ D. P. Craig and S. H. Walmsley, Excitons in Molecular Crystals (Benjamin, New York, 1968).

${ }^{24}$ H. Sternlicht, G. C. Nieman, and G. W. Robinson, J. Chem. Phys. 38, $1326(1963)$.

${ }^{25}$ H-K Hong and R. Kopelman, J. Chem. Phys. 55, 724 (1971).

${ }^{26}$ R. Kopelman, E. M. Monberg, and F. W. Ochs, Chem. Phys. 21, 373 (1977).

${ }^{27}$ U. Doberer, H. Port, and H. Benk, Chem. Phys. Lett. 85, 253 (1982).

${ }^{28}$ E. M. Monberg and R. Kopelman, Mol. Cryst. Liq. Cryst. 57, 271 (1980).

${ }^{29}$ G. W. Robinson and R. P. Frosch, J. Chem. Phys. 37, 1962 (1962).

${ }^{30}$ H. Stehfest, Commun. ACM 13, 47 (1970); 13, 624 (1970).

${ }^{31}$ R. C. Powell, J. Chem. Phys. 58, 920 (1973).

${ }^{32}$ P. Argyrakis and R. Kopelman, Chem. Phys. 78, 251 (1983).

${ }^{33} \mathrm{G}$. Zumofen and A. Blumen, Chem. Phys. Lett. 88, 63 (1982).

${ }^{34} \mathrm{~K}$. von Burg, L. Altwegg, and I. Zschokke-Gränacher, Phys. Rev. B 22, $2037(1980)$
${ }^{35}$ P. Argyrakis, D. Hooper, and R. Kopelman, J. Phys. Chem. 87, 1467 (1983).

${ }^{36}$ (a) A. Blumen and J. Manz, J. Chem. Phys. 71, 4694 (1979); (b) A. Blumen, ibid. 72, 2632 (1980).

${ }^{37}$ R. F. Loring, H. C. Andersen, and M. D. Fayer, Phys. Rev. Lett. 50, 1324 (1983).

${ }^{38}$ R. F. Loring, H. C. Andersen, and M. D. Fayer (preprint).

${ }^{39}$ R. F. Loring (private communication, 1982).

${ }^{40}$ M. E. Fisher and J. W. Essam, J. Math. Phys. 2, 609 (1961).

${ }^{41} \mathrm{~J}$. W. Essam, Rep. Prog. Phys. 43, 833 (1980).

${ }^{42}$ S. T. Gentry and R. Kopelman, J. Chem. Phys. 78, 373 (1983); J. Phys. Chem. (in press).

${ }^{43}$ R. F. Loring and M. D. Fayer, Chem. Phys. 70, 139 (1982).

${ }^{44}$ D. C. Ahlgren and R. Kopelman, Chem. Phys. Lett. 77, 135 (1981).

${ }^{45}$ D. C. Ahlgren, Doctoral dissertation, The University of Michigan, 1979.

${ }^{46}$ (a) P. W. Klymko and R. Kopelman, J. Phys. Chem. 86, 3686 (1982); (b) P. W. Klymko and R. Kopelman, ibid. 87, 4565 (1983).

${ }^{47} \mathrm{P}$. W. Klymko, Doctoral dissertation, The University of Michigan, 1983.

${ }^{48}$ R. Parson and R. Kopelman, J. Chem. Phys. 79, 1444 (1983).

${ }^{49}$ H. Haken and G. Strobl, Z. Phys. 262, 135 (1973).

${ }^{50}$ R. Parson, Chem. Phys. Lett. 99, 213 (1983).

${ }^{51}$ R. Kopelman, in Excited States II, edited by E. C. Lim (Academic, New York, 1975), p. 33.

${ }^{52} \mathbf{R}$. Kopelman, in Radiationless Processes in Molecules and Condensed Phases, Topics in Applied Physics Vol. 15, edited by F. K. Fong (Springer, Berlin, 1976), p. 297.

${ }^{53}$ A. H. Francis and R. Kopelman, in Laser Spectroscopy of Solids, Topics in Applied Physics Vol. 49, edited by W. M. Yen and P. M. Selzer (Springer, Berlin, 1981), p. 294.

${ }^{54}$ For example, S. K. Ma, Modern Theory of Critical Phenomena (Benjamin, New York, 1976).

${ }^{55}$ R. Loring, H. C. Andersen, and M. D. Fayer, J. Chem. Phys. 85, 149 (1984).

${ }^{56} \mathrm{~S}$. T. Gentry and K. Kopelman, J. Phys. Chem. (to be published). 